\title{
PHRAGMÉN-LINDELÖF THEOREM IN A COHOMOLOGICAL FORM
}

\author{
CHING-HER LIN ${ }^{1}$
}

\begin{abstract}
The main result of this paper is as follows. Given functions $\phi_{1}(\varepsilon), \ldots, \phi_{\nu}(\varepsilon)$ which are holomorphic in sectors $S_{1}, \ldots, S_{\nu}$, respectively, where $S_{1} \cup \cdots \cup S_{\nu}=\{\varepsilon$ : $|\arg \varepsilon|<\pi / 2 \alpha, 0<|\varepsilon|<\rho\}$ for $\alpha>1, \rho>0$, set $\phi_{j k}=\phi_{j}-\phi_{h}$ if $S_{j} \cap S_{h} \neq \varnothing$. Then $\left\{\phi_{j h}\right\}$ satisfy cocycle conditions $\phi_{j h}+\phi_{k l}=\phi_{j l}$ whenever $S_{j} \cap S_{k} \cap S_{l} \neq \varnothing$. In addition to the conditions $\left|\phi_{1}\right|<M_{0}$ and $\left|\phi_{\nu}\right|<M_{0}$ on the two rays of the boundary (i.e. $\arg \varepsilon=\pi / 2 \alpha$ ), and $\left|\phi_{j}(\varepsilon)\right| \leqslant A \exp (c /|\varepsilon|)$ in $S_{j}$ for some positive numbers $A$ and $c, j=1,2, \ldots, \nu$, if the $\left\{\phi_{j}\right\}$ satisfy the conditions $\left|\phi_{j k}\right|<M_{0}$ on $S_{j} \cap S_{h}(\neq \varnothing)$, then we get $\left|\phi_{j}\right|<M$ on $S_{j}, j=1,2, \ldots, \nu$. (From the cohomological point of view, we can get global results for $\phi_{j}$, once the local data on cocycles is known.)
\end{abstract}

1. Introduction. Let $\Omega=\{\varepsilon:-\pi / 2 \alpha<\arg \varepsilon<\pi / 2 \alpha, 0<|\varepsilon|<\rho\}$ be a sector in the right half complex $\varepsilon$-plane, where $\alpha>1$ and $\rho$ is a positive number. Let $f$ be a complex valued function which is continuous on $\Omega^{*}=\{\varepsilon:-\pi / 2 \alpha \leqslant \arg \varepsilon \leqslant \pi / 2 \alpha$, $0<|\varepsilon| \leqslant \rho\}$, holomorphic in $\Omega$, and there are positive constants $M$ and $c$ such that $|f(\varepsilon)| \leqslant M \exp (c /|\varepsilon|)$ for all $\varepsilon \in \Omega$. Assume, furthermore, that $|f(\varepsilon)| \leqslant M$ for all $\varepsilon$ on the boundary of $\Omega$. Then, the Phragmén-Lindelöf theorem states that $|f(\varepsilon)| \leqslant M$ for all $\varepsilon$ in $\Omega$ (see [2, p. 282]). In this paper, we shall generalize this theorem in a cohomological form; i.e. given functions $\phi_{1}(\varepsilon), \ldots, \phi_{\nu}(\varepsilon)$ which are holomorphic in sectors $S_{1}, \ldots, S_{\nu}$, respectively, $\Omega=S_{1} \cup \cdots \cup S_{\nu}$, set $\phi_{j k}=\phi_{j}-\phi_{k}$ if $S_{j} \cap S_{k} \neq \varnothing$. Then $\left\{\phi_{j k}\right\}$ satisfy cocycle conditions $\phi_{j k}+\phi_{k l}=\phi_{j l}$ whenever $S_{j} \cap S_{k} \cap S_{l} \neq \varnothing$. With this property, our theorem can be stated in the following way: If the $\left\{\phi_{j}\right\}$ satisfy the conditions $\left|\phi_{j k}\right| \leqslant M_{0}$ on $S_{j} \cap S_{k}(\neq \varnothing)$ in addition to the conditions $\left|\phi_{1}\right| \leqslant M_{0}$ and $\left|\phi_{\nu}\right| \leqslant M_{0}$ on the two rays of the boundary (i.e. $\arg \varepsilon= \pm \pi / 2 \alpha$ ), then we get $\left|\phi_{j}\right| \leqslant M$ on $S_{j}, j=1, \ldots, \nu$ (cf. Theorem 1). From the cohomological point of view, we can get global results for $\phi_{j}$ once the local data on cocycles is known. In our theorem, we have chosen those covering sectors $S_{1}, \ldots, S_{\nu}$ in a nice situation in which $S_{j} \cap S_{k} \neq \varnothing$ only for $k=j-1$ or $j+1, j=2, \ldots, \nu-1$ (cf. (2.1)). The following example will give a prototype of our result.

Let us consider two sectors $S_{j}=\left\{\varepsilon: a_{j}<\arg \varepsilon<b_{j}, 0<|\varepsilon|<\rho\right\}(j=1,2)$ where $-\pi / 2 \alpha=a_{1}<a_{2}<b_{1}<b_{2}=\pi / 2 \alpha$. Note that $S_{1} \cup S_{2}=\{\varepsilon:-\pi / 2 \alpha<\arg \varepsilon<$ $\pi / 2 \alpha, 0<|\varepsilon|<\rho\}$ and $S_{1} \cap S_{2} \neq \varnothing$ (cf. Figure 1).

Received by the editors December 9, 1982.

1980 Mathematics Subject Classification. Primary 30A10; Secondary 34E20.

Key words and phrases. Asymptotic theory, inequalities in the complex domain.

'This work was partially sponsored by the National Scierce Foundation under grant MCS 79-01998. This work was also a part of the author's dissertation for a Ph.D of Mathematics at the University of Minnesota, written under the supervision of Professor Sibuya. 


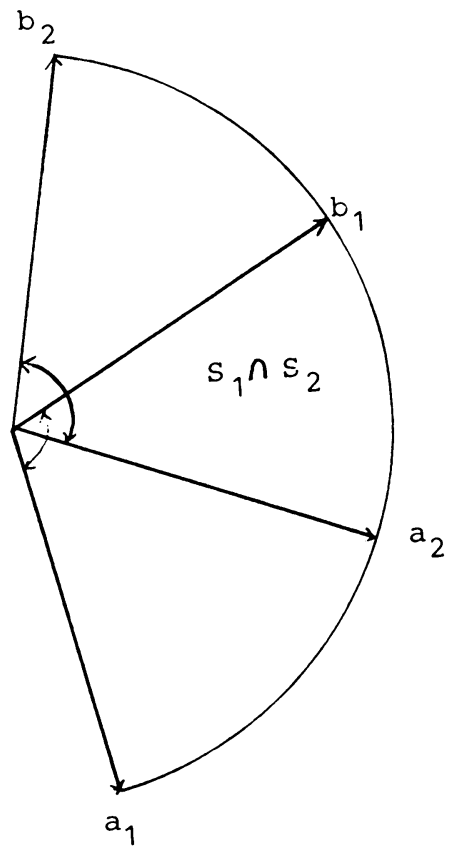

FigURE 1

Let $\phi_{j}$ be functions of $\varepsilon$ which are, respectively,

(1) holomorphic in $S_{j}$,

(2) continuous on $S_{j}^{*}=\left\{\varepsilon: a_{j} \leqslant \arg \varepsilon \leqslant b_{j}, 0<|\varepsilon| \leqslant \rho\right\}$, and

(3) $\left|\phi_{j}(\varepsilon)\right| \leqslant A \exp (c /|\varepsilon|)$ in $S_{j}$ for some positive numbers $c$ and $A$.

Assume that $\left|\phi_{2}(\varepsilon)-\phi_{1}(\varepsilon)\right| \leqslant M_{0}$ in $S_{1} \cap S_{2},\left|\phi_{1}(\varepsilon)\right| \leqslant M_{0}$ on the line segment $\arg \varepsilon=-\pi / 2 \alpha, 0<|\varepsilon|<\rho$, and $\left|\phi_{2}(\varepsilon)\right| \leqslant M_{0}$ on the line segment $\arg \varepsilon=\pi / 2 \alpha$, $0<|\varepsilon|<\rho$, for some $M_{0}$. Then we claim that $\left|\phi_{j}(\varepsilon)\right| \leqslant M$ in $S_{j}(j=1,2)$, respectively, for some positive number $M$ (cf. Theorem 1).

The difficulty in proving this result is due to the fact that $\phi_{1}$ and $\phi_{2}$ are two different functions in $S_{1} \cap S_{2}$, and hence a straightforward application of the Phragmén-Lindelöf theorem does not work.

The proof of this result, may be obtained by defining an auxiliary function (as in the proof of the Phragmén-Lindelöf theorem) together with Theorem 2 (cf. §2). Previously, Y. Sibuya [3] proved a result similar to Theorem 2, in the case when $S_{1} \cup S_{2} \cup \cdots \cup S_{\nu}$ is a disk. However, he did not show that $M$ was actually independent of $c_{1}$ as $c_{1}$ tends to zero. In the proof of Theorem 1, we shall let $c_{1}$ of Theorem 2 tend to zero (as in the proof of the Phragmén-Lindelöf theorem). Since $M$ in Theorem 2 is independent of $c_{1}$ as $c_{1}$ tends to zero, such a process will work. Notice also that if $\cup_{j=1}^{\nu} S_{j}$ is a disc, there are no boundary rays. In this paper, $\cup_{j=1}^{\nu} S_{j}$ being a sector, the analysis near the boundary rays becomes another additional difficulty (cf. Proof of Theorem 2 in $\S 3$ ).

Given functions $\delta_{1}(\varepsilon), \ldots, \delta_{\nu}(\varepsilon)$ which are holomorphic in sectors $S_{1}, \ldots, S_{\nu}$, respectively, $S_{1} \cup \cdots \cup S_{\nu}=\{\varepsilon:|\arg \varepsilon|<\pi / 2 \alpha, 0<|\varepsilon|<\rho\}$, satisfying certain reasonable assumptions and having relatively poor estimates on the two rays of the 
boundary (i.e. $\arg \varepsilon= \pm \pi / 2 \alpha$ ) which are assumed to be close to the imaginary axis, we can substantially improve such estimates along the real axis utilizing Theorem 3 (cf. Theorem 3 in $\$ 2$ ). The usefulness of our results is due to the fact that, in general, it is very difficult to get a global result all at once. In some cases where the Phragmén-Lindelöf theorem cannot be applied directly, we still can obtain a global result by putting suitable local results together through an application of our theorem (cf. e.g. [1 and 3]).

\section{The statement of theorems.}

THEOREM 1. Let $S_{j}=\left\{\varepsilon: a_{j}<\arg \varepsilon<b_{j}, 0<|\varepsilon|<\rho\right\}(j=1, \ldots, \nu)$ be sectors in the right half complex $\varepsilon$-plane where $\rho>0$ :

$$
-\pi / 2 \alpha=a_{1}<a_{2}<b_{1}<a_{3}<b_{2}<a_{4}<b_{3}<\cdots<a_{\nu}<b_{\nu-1}<b_{\nu}=\pi / 2 \alpha,
$$

$$
\alpha>1 \text {. }
$$

Let $\phi_{1}(\varepsilon), \ldots, \phi_{\nu}(\varepsilon)$ be functions of $\varepsilon$. Assume that

(1) $\phi_{j}(\varepsilon)$ is holomorphic in $S_{j}$ and continuous on $S_{j}^{*}=\left\{\varepsilon: a_{j} \leqslant \arg \varepsilon \leqslant b_{j}, 0<|\varepsilon| \leqslant\right.$ $\rho\}$,

(2) $\left|\phi_{j}(\varepsilon)\right| \leqslant A \exp (c /|\varepsilon|)$ in $S_{j}$, for some positive numbers $A$ and $c$,

(3) $\left|\phi_{j+1}(\varepsilon)-\phi_{j}(\varepsilon)\right|<M_{0}$ in $S_{j} \cap S_{j+1},\left|\phi_{1}(\varepsilon)\right| \leqslant M_{0}$ on the line segment $\arg \varepsilon=$ $-\pi / 2 \alpha, 0<|\varepsilon|<\rho$, and $\left|\phi_{\nu}(\varepsilon)\right| \leqslant M_{0}$ on the line segment arg $\varepsilon=\pi / 2 \alpha, 0<|\varepsilon|<\rho$, for some positive number $M_{0}$. Then, there exists a positive number $M$ such that $\left|\phi_{j}(\varepsilon)\right| \leqslant M$ in $S_{j}, j=1, \ldots, \nu$.

REMARK. The inequalities (2.1) mean that $S_{j} \cap S_{k} \neq \varnothing$ only for $k=j-1$ or $j+1, j=2,3, \ldots, \nu-1$.

TheOREM 2. Let $S_{j}=\left\{\varepsilon: a_{j}<\arg \varepsilon<b_{j}, 0<|\varepsilon|<\rho\right\}(j=1,2, \ldots, \nu)$ be sectors in the complex $\varepsilon$-plane, where $\rho>0$ :

$$
-\pi<a_{1}<a_{2}<b_{1}<a_{3}<b_{2}<a_{4}<b_{3}<\cdots<a_{\nu}<b_{\nu-1}<b_{\nu}<\pi .
$$

Let $\delta_{1}(\varepsilon), \ldots, \delta_{\nu}(\varepsilon)$ be functions of $\varepsilon$. Assume that

(1) $\delta_{j}(\varepsilon)$ is holomorphic in $S_{j}$, continuous on $S_{j}^{*}$,

(2) $\delta_{j}(\varepsilon)$ is asymptotically zero as $\varepsilon$ tends to zero in $S_{j}$, i.e. $\left|\delta_{j}(\varepsilon)\right| \leqslant K_{N}|\varepsilon|^{N}$ $(N=0,1,2, \ldots)$ in $S_{j}$ for some positive numbers $K_{N}$,

(3) $\left|\delta_{j+1}(\varepsilon)-\delta_{j}(\varepsilon)\right| \leqslant M_{0} \exp \left(-c_{1} /|\varepsilon|^{\lambda}\right)$ in $S_{j} \cap S_{j+1},\left|\delta_{1}(\varepsilon)\right| \leqslant M_{0} \exp \left(-c_{1} /|\varepsilon|^{\lambda}\right)$ on the line segment $\arg \varepsilon=a_{1}, 0<|\varepsilon|<\rho$, and $\left|\delta_{\nu}(\varepsilon)\right| \leqslant M_{0} \exp \left(-c_{1} /|\varepsilon|^{\lambda}\right)$ on the line segment $\arg \varepsilon=b_{\nu}, 0<|\varepsilon|<\rho$, for some positive numbers $c_{1}, M_{0}$ and $\lambda$. Then, there exists a positive number $M$ which is independent of $c_{1}$ as $c_{1}$ tends to zero such that

$$
\left|\delta_{j}(\varepsilon)\right| \leqslant M \exp \left(-c_{1} /|\varepsilon|^{\lambda}\right) \quad \text { in } S_{j}, j=1,2, \ldots, \nu .
$$

THEOREM 3. Let $S_{j}=\left\{\varepsilon: a_{j}<\arg \varepsilon<b_{j}, 0<|\varepsilon|<\rho\right\}$ be sectors in the right half complex $\varepsilon$-plane, where $\rho>0$ :

$-\pi / 2 \alpha=a_{1}<a_{2}<b_{1}<a_{3}<b_{2}<a_{4}<b_{3}<\cdots<a_{\nu}<b_{\nu-1}<b_{\nu}=\pi / 2 \alpha$, 
Let $\delta_{1}(\varepsilon), \ldots, \delta_{\nu}(\varepsilon)$ be functions of $\varepsilon$. Assume that

(1) $\delta_{j}(\varepsilon)$ is holomorphic in $S_{j}$, continuous on $S_{j}^{*}$,

(2) $\delta_{j}(\varepsilon)$ is asymptotically zero, as $\varepsilon$ tends to zero in $S_{j}$,

(3) $\left|\delta_{j+1}(\varepsilon)-\delta_{j}(\varepsilon)\right| \leqslant M_{0} \exp \{-\mu \operatorname{Re}(1 / \varepsilon)\}$ in $S_{j} \cap S_{j+1}$,

$$
\left|\delta_{1}(\varepsilon)\right| \leqslant M_{0} \exp \{-\mu \operatorname{Re}(1 / \varepsilon)\}
$$

on the line segment $\arg \varepsilon=-\pi / 2 \alpha, 0<|\varepsilon|<\rho$, and

$$
\left|\delta_{\nu}(\varepsilon)\right| \leqslant M_{0} \exp \{-\mu \operatorname{Re}(1 / \varepsilon)\}
$$

on the line segment $\arg \varepsilon=\pi / 2 \alpha, 0<|\varepsilon|<\rho$, for some positive numbers $\mu$ and $M_{0}$. Then, there exists a positive number $M$ such that $\left|\delta_{j}(\varepsilon)\right| \leqslant M \exp \{-\mu \operatorname{Re}(1 / \varepsilon)\}$ in $S_{j}$, $j=1,2, \ldots, \nu$.

\section{Proof of the theorems.}

(1) Proof of Theorem 2. We denote by $V_{j}$ the intersections $S_{j} \cap S_{j+1}, j=$ $1,2, \ldots, \nu-1$, respectively, and consider an open sector

$$
S=\left\{\varepsilon: a_{1}<\arg \varepsilon<b_{\nu}, 0<|\varepsilon|<\rho_{0}\right\}, \text { where } 0<\rho_{0}<\rho .
$$

We choose $\nu-1$ line segments $l_{1}, l_{2}, \ldots, l_{\nu-1}$ such that $l_{j} \subset V_{j}$, i.e. $l_{j}: \varepsilon=t e^{i \alpha}$ $\left(0<t<\rho_{0}\right.$, for some $\alpha_{j}$ in $\left.\left(a_{j+1}, b_{j}\right)\right)$. These $\nu-1$ line segments divide the open sector $S$ (cf. (3.1)) into $\nu$ open sectors $\hat{S}_{1}, \hat{S}_{2}, \ldots, \hat{S}_{\nu}$ (cf. Figure 2 ).

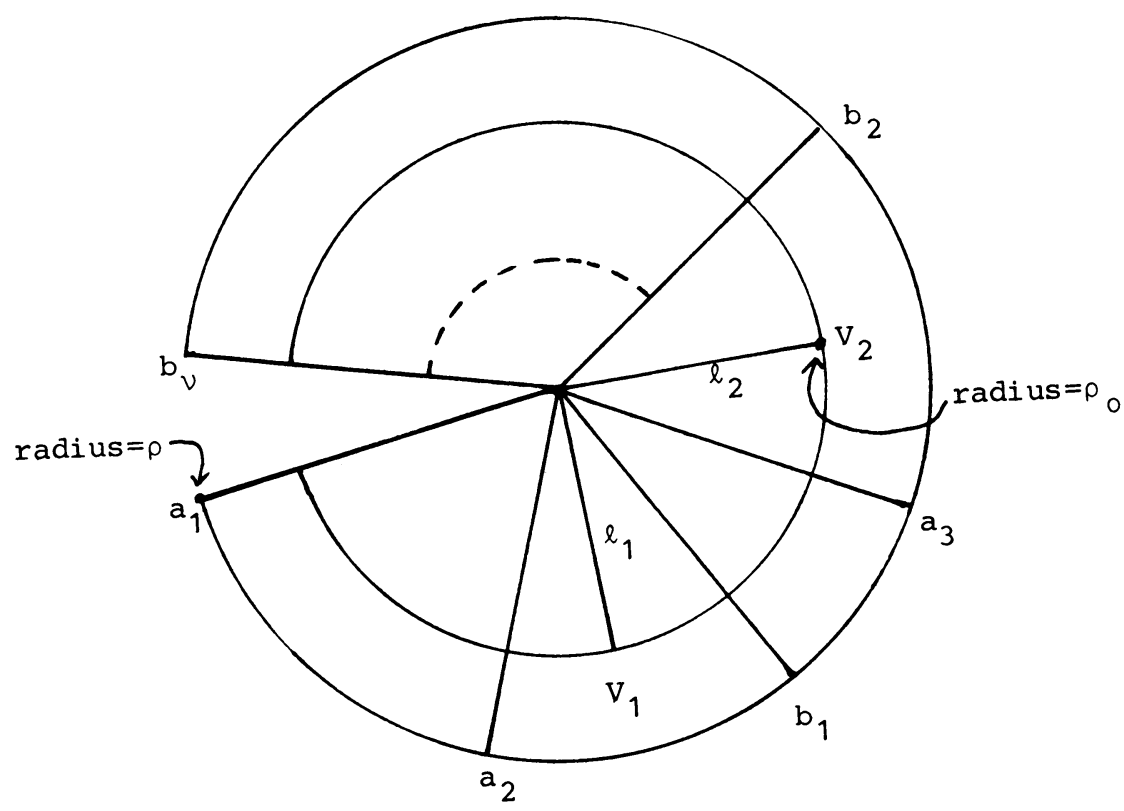

FIGURE 2 
Let $\gamma_{j}(j=1,2, \ldots, \nu)$ be the circular arcs which are defined by $\varepsilon=\rho_{0} e^{i \xi}\left(\alpha_{j-1} \leqslant\right.$ $\left.\xi \leqslant \alpha_{j}\right)$, respectively, where $\alpha_{0}=a_{1}, \alpha_{\nu}=b_{\nu}$. Set, $l_{0}: \varepsilon=t e^{i a_{1}}\left(0<t<\rho_{0}\right), l_{\nu}$ : $\varepsilon=t e^{i h_{\nu}}\left(0<t<\rho_{0}\right)$. Then $\gamma_{1}+\gamma_{2}+\cdots+\gamma_{\nu}=C=\left\{\varepsilon:|\varepsilon|=\rho_{0}, a_{1} \leqslant \arg \varepsilon \leqslant b_{\nu}\right\}$. The boundaries of $\hat{S}_{1}, \hat{S}_{2}, \ldots, \hat{S}_{\nu}$ are, respectively, $l_{j-1}+\gamma_{j}-l_{j}, j=1,2, \ldots, \nu$.

For $\varepsilon \in \hat{S}_{1} \cup \hat{S}_{2} \cup \cdots \cup \hat{S}_{\nu}$, set $\delta(\varepsilon)=\delta_{j}(\varepsilon)$ if $\varepsilon \in \hat{S}_{j}$. Since

$$
\frac{1}{2 \pi i} \int_{l_{j-1}+\gamma_{j}-l_{j}} \frac{\delta_{j}(\xi)}{\xi-\varepsilon} d \xi= \begin{cases}\delta_{j}(\varepsilon), & \varepsilon \in \hat{S}_{j}, \\ 0, & \varepsilon \notin \hat{S}_{j},\end{cases}
$$

we have

$$
\delta(\varepsilon)=\frac{1}{2 \pi i} \sum_{j=1}^{\nu} \int_{l_{j-1}+\gamma_{j}-l_{j}} \frac{\delta_{j}(\xi)}{\xi-\varepsilon} d \xi \quad \text { in } \hat{S}_{1} \cup \hat{S}_{2} \cup \cdots \cup \hat{S}_{\nu}
$$

Utilizing $1 /(\xi-\varepsilon)=\sum_{m=0}^{N} \xi^{-(m+1)} \varepsilon^{m}+\varepsilon^{N+1} / \xi^{N+1}(\xi-\varepsilon)$, we derive

$$
\begin{aligned}
\delta(\varepsilon)= & \frac{1}{2 \pi i} \sum_{m=0}^{N}\left\{\sum_{j=1}^{\nu} \int_{l_{j-1}+\gamma_{j}-l_{j}} \xi^{-(m+1)} \delta_{j}(\xi) d \xi\right\} \varepsilon^{m} \\
& +\left\{\frac{1}{2 \pi i} \sum_{j=1}^{\nu} \int_{l_{j-1}+\gamma_{j}-l_{j}} \frac{\delta_{j}(\xi)}{\xi^{N+1}(\xi-\varepsilon)} d \xi\right\} \varepsilon^{N+1} .
\end{aligned}
$$

Since $\delta(\varepsilon)$ is asymptotically zero as $\varepsilon$ tends to zero in $\hat{S}_{1} \cup \hat{S}_{2} \cup \cdots \cup \hat{S}_{\nu}$, the first term of (3.2) must be zero. Hence

$$
\delta(\varepsilon)=\left\{\frac{1}{2 \pi i} \sum_{j=1}^{\nu} \int_{l_{j-1}+\gamma_{j}-l_{j}} \frac{\delta_{j}(\xi)}{\xi^{N+1}(\xi-\varepsilon)} d \xi\right\} \varepsilon^{N+1} .
$$

Therefore, we arrive at the following formula:

$$
\begin{aligned}
\delta(\varepsilon)=\frac{1}{2 \pi i}\left\{\int_{l_{0}} \frac{\delta_{1}(\xi)}{\xi^{N}(\xi-\varepsilon)} d \xi-\int_{l_{\nu}} \frac{\delta_{\nu}(\xi)}{\xi^{N}(\xi-\varepsilon)} d \xi\right. \\
\left.\quad+\sum_{j=1}^{\nu-1} \int_{l_{j}} \frac{\sigma_{j}(\xi)}{\xi^{N}(\xi-\varepsilon)} d \xi+\int_{C} \frac{\delta(\xi)}{\xi^{N}(\xi-\varepsilon)} d \xi\right\} \varepsilon^{N}
\end{aligned}
$$

for $\varepsilon \in \hat{S}_{1} \cup \hat{S}_{2} \cup \cdots \cup \hat{S}_{\nu}$ and $N=1,2, \ldots$ where $\sigma_{j}=\delta_{j+1}-\delta_{j}$. Construct $\nu$ open sectors $\tilde{S}_{1}, \tilde{S}_{2}, \ldots, \tilde{S}_{\nu}$ such that the boundaries of $\tilde{S}_{j}$ are $\Omega_{j} \cup T_{j} \cup \Omega^{j}$, where

$$
\begin{gathered}
\Omega_{j}=\left\{\varepsilon: \varepsilon=|\varepsilon| e^{i\left(\alpha_{j-1}+\theta\right)},|\varepsilon| \leqslant \rho_{1}\right\}, \\
\Omega^{j}=\left\{\varepsilon: \varepsilon=|\varepsilon| e^{i\left(\alpha_{j}-\theta\right)},|\varepsilon| \leqslant \rho_{1}\right\}, \\
T_{j}=\left\{\varepsilon: \varepsilon=\rho_{1} e^{i \beta}, \alpha_{j-1}+\theta \leqslant \beta \leqslant \alpha_{j}-\theta\right\}, \quad j=1,2, \ldots, \nu,
\end{gathered}
$$

$0<\rho_{1}<\rho_{0}$ and $\theta$ is a small positive number such that $\lambda \theta<\pi$ (cf. Figure 3). 


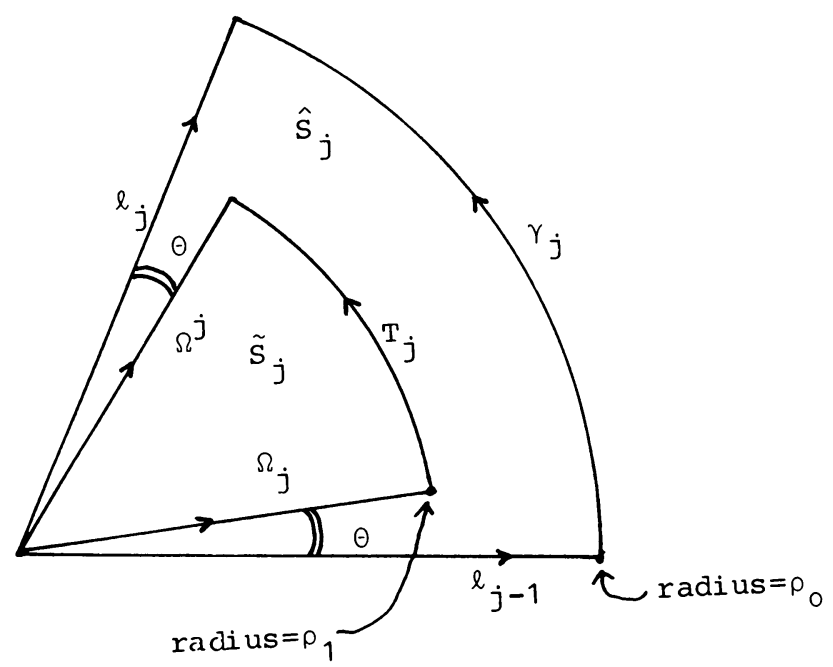

FIGURE 3

Then, $\tilde{S}_{j} \subset \hat{S}_{j}, j=1,2, \ldots, \nu$.

For $\varepsilon \in \tilde{S}_{1} \cup \tilde{S}_{2} \cup \cdots \cup \tilde{S_{\nu}}$,

$$
\begin{aligned}
\left|\int_{C} \frac{\delta(\xi)}{\xi^{N}(\xi-\varepsilon)} d \xi\right| & \leqslant \int_{C} \frac{|\delta(\xi)|}{|\xi|^{N}|\xi-\varepsilon|}|d \xi| \leqslant \int_{C} \frac{K_{\delta}}{\rho_{0}^{N}\left(\rho_{0}-\rho_{1}\right)}|d \xi| \\
& =\frac{K_{\delta} \rho_{0}}{\rho_{0}^{N}\left(\rho_{0}-\rho_{1}\right)}\left(b_{\nu}-a_{1}\right)=\frac{B_{\delta}}{\rho_{0}^{N-1}}
\end{aligned}
$$

where $K_{\delta}=\max _{\xi \in C}|\delta(\xi)|, B_{\delta}=K_{\delta}\left(b_{\nu}-a_{1}\right) /\left(\rho_{0}-\rho_{1}\right)$.

$$
\begin{aligned}
\left|\int_{l_{0}} \frac{\delta_{1}(\xi)}{\xi^{N}(\xi-\varepsilon)} d \xi\right| & =\left|\int_{0}^{\rho_{0}} \frac{\delta_{1}\left(t e^{i a_{1}}\right)}{t^{N} e^{i N a_{1}}\left(t e^{i a_{1}}-\varepsilon\right)} e^{i a_{1}} d t\right| \leqslant \int_{0}^{\rho_{0}} \frac{\left|\delta_{1}\left(t e^{i a_{1}}\right)\right|}{t^{N+1} \sin \theta} d t \\
& \leqslant \frac{M_{0}}{\sin \theta} \int_{0}^{\rho_{0}} t^{-N-1} \exp \left(-c_{1} / t^{\lambda}\right) d t \\
& =\frac{M_{0}}{\lambda \sin \theta} \int_{1 / \rho_{0}^{\lambda}}^{+\infty} \tau^{(N / \lambda)-1} \exp \left(-c_{1} \tau\right) d \tau \\
& <\frac{M_{0}}{\lambda \sin \theta} \int_{0}^{+\infty} \tau^{(N / \lambda)-1} \exp \left(-c_{1} \tau\right) d \tau \\
& =\frac{M_{0}}{\lambda \sin \theta} C_{1}^{-(N / \lambda)} \Gamma(N / \lambda) .
\end{aligned}
$$

Similarly,

$$
\left|\int_{l_{\nu}} \frac{\delta_{\nu}(\xi)}{\xi^{N}(\xi-\varepsilon)} d \xi\right|<\frac{M_{0}}{\lambda \sin \theta} C_{1}^{-(N / \lambda)} \Gamma(N / \lambda)
$$


and

$$
\left|\int_{l_{j}} \frac{\sigma_{j}(\xi)}{\xi^{N}(\xi-\varepsilon)} d \xi\right|<\frac{M_{0}}{\lambda \sin \theta} C_{1}^{-(N / \lambda)} \Gamma(N / \lambda), \quad j=1,2, \ldots, \nu-1 .
$$

Since $\Gamma(N / \lambda) \leqslant M_{1}(N / \lambda)^{(N / \lambda)} e^{-(N / \lambda)}$ for some $M_{1}>0$, we have

$$
\begin{aligned}
& \left|\int_{l_{0}} \frac{\delta_{1}(\xi)}{\xi^{N}(\xi-\varepsilon)} d \xi\right|<\frac{M_{0} M_{1}}{\lambda \sin \theta}\left(\frac{N}{c_{1} \lambda}\right)^{(N / \lambda)} e^{-(N / \lambda)}, \\
& \left|\int_{l_{\nu}} \frac{\delta_{\nu}(\xi)}{\xi^{N}(\xi-\varepsilon)} d \xi\right|<\frac{M_{0} M_{1}}{\lambda \sin \theta}\left(\frac{N}{c_{1} \lambda}\right)^{(N / \lambda)} e^{-(N / \lambda)}
\end{aligned}
$$

and

$$
\left|\int_{l_{j}} \frac{\sigma_{j}(\xi)}{\xi^{N}(\xi-\varepsilon)} d \xi\right|<\frac{M_{0} M_{1}}{\lambda \sin \theta}\left(\frac{N}{c_{1} \lambda}\right)^{(N / \lambda)} e^{-(N / \lambda)}
$$

for $\varepsilon \in \tilde{S}_{1} \cup \cdots \cup \tilde{S_{\nu}}, j=1,2, \ldots, \nu-1$. Then,

$$
\begin{aligned}
|\delta(\varepsilon)| & \leqslant \frac{1}{2 \pi}\left\{\frac{(\nu+1) M_{0} M_{1}}{\lambda \sin \theta}\left(\frac{N}{c_{1} \lambda}\right)^{(N / \lambda)} e^{-(N / \lambda)}+\frac{B_{\delta}}{\rho_{0}^{N-1}}\right\}|\varepsilon|^{N} \\
& =\frac{M_{2}}{2 \pi}\left\{1+\frac{B_{\delta} \rho_{0}}{M_{2}}\left(\frac{c_{1} \lambda e}{N \rho_{0}^{\lambda}}\right)^{(N / \lambda)}\right\}\left(\frac{N|\varepsilon|^{\lambda}}{c_{1} \lambda}\right)^{(N / \lambda)} e^{-(N / \lambda)},
\end{aligned}
$$

where $M_{2}=(\nu+1) M_{0} M_{1} / \lambda \sin \theta>0$.

Since $\left\{1+\left(B_{\delta} \rho_{0} / M_{2}\right)\left(c_{1} \lambda e / N \rho_{0}^{\lambda}\right)^{(N / \lambda)}\right\} \rightarrow 1$ as $c_{1} \rightarrow 0$, we have

$$
|\delta(\varepsilon)| \leqslant M_{3}(\delta)\left(\frac{N|\varepsilon|^{\lambda}}{c_{1} \lambda}\right)^{(N / \lambda)} e^{-(N / \lambda)}
$$

for $\varepsilon \in \tilde{S}_{1} \cup \tilde{S_{2}} \cup \cdots \cup \tilde{S_{\nu}}$, where $M_{3}(\delta)$ is a constant which is independent of $c_{1}$ as $c_{1}$ tends to zero.

For a given $\varepsilon$, choose $N$ so that $N / \lambda<c_{1} /|\varepsilon|^{\lambda} \leqslant(N+1) / \lambda$. We have

$$
\frac{N|\varepsilon|^{\lambda}}{\lambda c_{1}}<1 \text { and }-\frac{c_{1}}{|\varepsilon|^{\lambda}} \geqslant-\frac{N+1}{\lambda} .
$$

Then, it follows from (3.3) and (3.4) that we have

$$
|\delta(\varepsilon)| \leqslant M_{3}(\delta) e^{1 / \lambda} \exp \left(-c_{1} /|\varepsilon|^{\lambda}\right) \leqslant M_{4} \exp \left(-c_{1} /|\varepsilon|^{\lambda}\right)
$$

where $M_{4}=\max \left\{M_{0}, M_{3}(\delta) e^{1 / \lambda}\right\}$ which is independent of $c_{1}$ as $c_{1}$ tends to zero. Choosing $l_{1}, l_{2}, \ldots, l_{\nu-1}$ in various ways, we can prove that

$$
\left|\delta_{j}(\varepsilon)\right| \leqslant M_{4} \exp \left(-c_{1} /|\varepsilon|^{\lambda}\right) \quad \text { in } S_{j}^{\prime}, j=2, \ldots, \nu-1,
$$


where

$$
S_{j}^{\prime}=\left\{\varepsilon: a_{j}<\arg \varepsilon<b_{j}, 0<|\varepsilon|<\rho_{1}\right\}, \quad j=1,2, \ldots, \nu .
$$

Since $l_{0}$ and $l_{\nu}$ are boundaries of $S$, they cannot be moved. To obtain a similar estimate in $S_{1}^{\prime}$, set $c_{2}=c_{1} / \cos (\lambda \theta / 2)$, then

$$
\left|\delta_{1}\left(\varepsilon e^{i\left(a_{1}+\theta / 2\right)}\right)\right| \leqslant M_{4} \exp \left(-c_{2} \cos (\lambda \theta / 2) /|\varepsilon|^{\lambda}\right)
$$

on

$$
\left\{\varepsilon: \varepsilon=|\varepsilon| e^{i \theta / 2}, 0<|\varepsilon|<\rho_{1}\right\} \cup\left\{\varepsilon: \varepsilon=|\varepsilon| e^{-i \theta / 2}, 0<|\varepsilon|<\rho_{1}\right\} .
$$

Let $h(\varepsilon)=\exp \left\{c_{2} / \varepsilon^{\lambda}\right\}\left(\varepsilon \in\left\{\varepsilon: 0<|\varepsilon| \leqslant \rho_{1}\right\}\right)$. For $\arg \varepsilon=\theta / 2$, arg $\varepsilon=-\theta / 2$, we have

$\left|\delta_{1}\left(\varepsilon e^{i\left(a_{1}+\theta / 2\right)}\right) h(\varepsilon)\right| \leqslant M_{4} \exp \left\{-c_{2} \cos (\lambda \theta / 2) /|\varepsilon|^{\lambda}\right\} \cdot \exp \left\{c_{2} \cos (\lambda \theta / 2) /|\varepsilon|^{\lambda}\right\}=M_{4}$ and for $\varepsilon=|\varepsilon| e^{i \eta},-\theta / 2<\eta<\theta / 2$, we have

$$
\left|\delta_{1}\left(\varepsilon e^{i\left(a_{1}+\theta / 2\right)}\right) h(\varepsilon)\right| \leqslant E \exp \left\{c_{2} \cos (\lambda \eta) /|\varepsilon|^{\lambda}\right\} \leqslant E \exp \left\{c_{2} /|\varepsilon|^{\lambda}\right\},
$$

where $E$ is a bound for $\delta_{1}\left(\varepsilon e^{i\left(a_{1}+\theta / 2\right)}\right)$ on $\left\{\varepsilon:-\theta / 2 \leqslant \arg \varepsilon \leqslant \theta / 2,0<|\varepsilon| \leqslant \rho_{1}\right\}$. Then, by the Phragmén-Lindelöf theorem, we have

$$
\left|\delta_{1}\left(\varepsilon e^{i\left(a_{1}+\theta / 2\right)}\right) h(\varepsilon)\right| \leqslant M_{4} \text { for } \varepsilon \in\left\{\varepsilon:-\theta / 2<\arg \varepsilon<\theta / 2,0<|\varepsilon|<\rho_{1}\right\}
$$

i.e. $\left|\delta_{1}\left(\varepsilon e^{i\left(a_{1}+\theta / 2\right)}\right)\right| \leqslant M_{4} \exp \left\{-c_{2} \cos (\lambda \theta / 2) /|\varepsilon|^{\lambda}\right\}$ for

$$
\varepsilon=|\varepsilon| e^{i \eta} \in\left\{\varepsilon:-\theta / 2<\arg \varepsilon<\theta / 2,0<|\varepsilon|<\rho_{1}\right\} .
$$

Thus, $\left|\delta_{1}(\varepsilon)\right| \leqslant M_{4} \exp \left(-c_{1} /|\varepsilon|^{\lambda}\right)$ in $S_{1}^{\prime}$; similarly, $\left|\delta_{\nu}(\varepsilon)\right| \leqslant M_{4} \exp \left(-c_{1} /|\varepsilon|^{\lambda}\right)$ in $S_{\nu}^{\prime}$. Choosing $M$ as the maximal value of $M_{4}$ and a bound of $\left|\delta_{j}(\varepsilon)\right| \exp \left(c_{1} / \rho_{1}^{\lambda}\right)$ on $\{\varepsilon$ : $\left.a_{j} \leqslant \arg \varepsilon \leqslant b_{j}, \rho_{1} \leqslant|\varepsilon| \leqslant \rho\right\}, j=1,2, \ldots, \nu$, which is independent of $c_{1}$ as $c_{1}$ tends to zero, we can obtain $\left|\delta_{j}(\varepsilon)\right| \leqslant M \exp \left(-c_{1} /|v \varepsilon|^{\mu}\right)$ in $S_{j}, j=1,2, \ldots, \nu$.

(2) Proof of Theorem 1. For each $\eta>0$ we define an auxiliary function

$$
h_{\eta}(\varepsilon)=\exp \left\{-\eta / \varepsilon^{\lambda}\right\} \quad(\varepsilon \in\{\varepsilon:-\pi / 2 \alpha \leqslant \arg \varepsilon \leqslant \pi / 2 \alpha, 0<|\varepsilon| \leqslant \rho\})
$$

where $1<\lambda<\alpha$. Set, $\delta_{j}(\varepsilon)=\phi_{j}(\varepsilon) h_{\eta}(\varepsilon)$ which depends on $\eta, j=1,2, \ldots, \nu$. Then, for $\varepsilon \in l_{0}$,

$$
\begin{aligned}
\left|\delta_{1}(\varepsilon)\right| & =\left|\phi_{1}(\varepsilon) h_{\eta}(\varepsilon)\right| \leqslant M_{0}\left|\exp \left\{-\eta /|\varepsilon|^{\lambda} e^{-\pi \lambda i / 2 \alpha}\right\}\right| \\
& =M_{0} \exp \left\{-\eta \cos (\lambda \pi / 2 \alpha) /|\varepsilon|^{\lambda}\right\}
\end{aligned}
$$

for $\varepsilon \in l_{\nu}$,

$$
\begin{aligned}
\left|\delta_{\nu}(\varepsilon)\right| & =\left|\phi_{\nu}(\varepsilon) h_{\eta}(\varepsilon)\right| \leqslant M_{0}\left|\exp \left\{-\eta /|\varepsilon|^{\lambda} e^{\pi \lambda i / 2 \alpha}\right\}\right| \\
& =M_{0} \exp \left\{-\eta \cos (\lambda \pi / 2 \alpha) /|\varepsilon|^{\lambda}\right\}
\end{aligned}
$$

and for $\varepsilon=|\varepsilon| e^{i \beta_{j}} \in S_{j} \cap S_{j+1}$,

$$
\left|\delta_{j+1}(\varepsilon)-\delta_{j}(\varepsilon)\right|=\left|\phi_{j+1}(\varepsilon)-\phi_{j}(\varepsilon)\right|\left|h_{\eta}(\varepsilon)\right| \leqslant M_{0} \exp \left\{-\eta \cos \left(\lambda\left|\beta_{j}\right|\right) /|\varepsilon|^{\lambda}\right\} .
$$


Since $0<\lambda\left|\beta_{j}\right|<\lambda \pi / 2 \alpha$, we have $\cos \left(\lambda\left|\beta_{j}\right|\right)>\cos (\lambda \pi / 2 \alpha)$. Then we derive

$$
\left|\delta_{j+1}(\varepsilon)-\delta_{j}(\varepsilon)\right| \leqslant M_{0} \exp \left\{-\eta \cos (\lambda \pi / 2 \alpha) /|\varepsilon|^{\lambda}\right\} \quad \text { in } S_{j} \cap S_{j+1},
$$

and $\delta_{j}(\varepsilon)$ is asymptotically zero as $\varepsilon$ tends to zero in $S_{j}$. Set $c_{1}=\eta \cos (\lambda \pi / 2 \alpha)$. Then by Theorem 2, there exists a positive number $H$ which is independent of $c_{1}$ as $c_{1}$ tends to zero $\left(c_{1} \rightarrow 0\right.$, as $\left.\eta \rightarrow 0\right)$ such that

$$
\left|\delta_{j}(\varepsilon)\right|=\left|\phi_{j}(\varepsilon) h_{\eta}(\varepsilon)\right| \leqslant M \exp \left\{-\eta \cos (\lambda \pi / 2 \alpha) /|\varepsilon|^{\lambda}\right\} \quad \text { in } S_{j}, j=1,2, \ldots, \nu .
$$

As $\eta \rightarrow 0, h_{\eta}(\varepsilon) \rightarrow 1$ for every $\varepsilon$; so we conclude that $\left|\phi_{j}(\varepsilon)\right| \leqslant M$ in $S_{j}, j=1,2, \ldots, \nu$.

(3) Proof of Theorem 3. Let $h(\varepsilon)=\exp \{\mu / \varepsilon\}, \varepsilon \in S=\{\varepsilon:-\pi / 2 \alpha \leqslant \arg \varepsilon \leqslant$ $\pi / 2 \alpha, 0<|\varepsilon| \leqslant \rho\}$, and set $\phi_{j}(\varepsilon)=\delta_{j}(\varepsilon) h(\varepsilon), j=1,2, \ldots, \nu$. Then, for $\varepsilon=|\varepsilon| e^{-\pi i / 2 \alpha}$,

$$
\left|\phi_{1}(\varepsilon)\right|=\left|\delta_{1}(\varepsilon)\right||h(\varepsilon)| \leqslant M_{0} \exp \{-\mu \operatorname{Re}(1 / \varepsilon)\} \exp \{\mu \operatorname{Re}(1 / \varepsilon)\}=M_{0} ;
$$

similarly, for $\varepsilon=|\varepsilon| e^{\pi i / 2 \alpha},\left|\phi_{\nu}(\varepsilon)\right| \leqslant M_{0}$; for $\varepsilon=|\varepsilon| e^{i \beta_{j}} \in S_{j} \cap S_{j+1}, \mid \phi_{j+1}(\varepsilon)-$ $\phi_{j}(\varepsilon) \mid \leqslant M_{0}$; and for $\varepsilon=|\varepsilon| e^{i \xi_{j}} \in S_{j}$,

$$
\left|\phi_{j}(\varepsilon)\right|=\left|\delta_{j}(\varepsilon)\right||h(\varepsilon)| \leqslant A \exp \left\{\mu \cos \xi_{j} /|\varepsilon|\right\} \leqslant A \exp \{\mu /|\varepsilon|\}
$$

where $A>0$ is a bound for $\delta_{j}$ in $S_{j}$. By Theorem 1 , we have $\left|\phi_{j}(\varepsilon)\right| \leqslant M$ in $S_{j}$ $(j=1,2, \ldots, \nu)$, i.e. $\left|\delta_{j}(\varepsilon)\right| \leqslant M \exp \{-\mu \operatorname{Re}(1 / \varepsilon)\}$ in $S_{j}, j=1,2, \ldots, \nu$.

AcKnowledgement. I would like to thank my advisor Professor Sibuya for his many valuable suggestions.

\section{REFERENCES}

1. C.-H. Lin. The sufficiency of Matkowsky-condition in the problem of resonance, Trans. Amer. Math. Soc. (to appear).

2. W. Rudin, Real and complex analysis, McGraw-Hill, New York, 1974.

3. Y. Sibuya, $A$ theorem concerning uniform simplification at a transition point and the problem of resonance, SIAM J. Math. Anal. 12 (1981), 653-668.

Department of Mathematics, College of Natural Science, Fujen University, Hsin Chuang 242, TAIPEI, TAIWAN 